\title{
INTERACTION OF SH-WAVES WITH A FINITE CRACK IN A HALF-SPACE
}

\author{
J. Y. HUANG \\ Aeronautical Research Laboratory, Aero Industry Development Center, P.O. Box 90008-11-3, \\ Taichung, Taiwan, R.O.C.
}

\begin{abstract}
The diffraction of time harmonic antiplane shear waves by a finite length crack embedded in a half-space is considered. Based on the qualitatively similar features of cracks and dislocations, with the aid of image method, the dislocation density function as well as the stress field due to such dislocations are expressed by a system of singular integral equations. These equations with kernels containing Bessel functions can be solved by Galerkin numerical scheme. As the crack is nearly in contact with the free surface, the problem can be regarded as the diffraction of elastic waves by an edge crack. The difference between numerical solutions for two types of boundary conditions, free of traction and clamped surface, is examined. Graphical results for the dynamic stress intensity factors as functions of waves number, angle of incidence and position of the crack are presented.
\end{abstract}

\section{INTRODUCTION}

IT HAS been recognized that horizontally polarized shear waves (SH-waves) which are diffracted by the crack can give rise to high elevation of local stresses. The dynamic stress intensity factors, which may be about $30 \%$ higher than the corresponding static ones, depend on the crack size and the wavelength or frequence of the travelling waves [1]. Mal [2] made detailed studies on the stress field in the vicinity of a crack due to an incident anti-plane shear wave. Jain and Kanwal [3] and Itou [4] presented a solution for the problem of diffraction of normally incident anti-plane shear waves by two asymmetrical stationary coplanar cracks located in an infinite isotropic medium. Takakuda [5] obtained the stress intensity factors of two nonplanar parallel cracks. The effects of SH, P and SV waves on the stress intensity factors of two cracks at arbitrary positions in an infinite isotropic elastic medium had been analysed by means of a dislocation model developed by Huang and So [6-8].

Since attempts to analyse stress intensity factors of a crack with boundaries poses difficulties in analytical treatment, no wide efforts have concentrated on the elastodynamic response of a crack with its neighboring boundaries. Datta [9] obtained an approximate solution for the diffraction of $\mathrm{SH}$-waves at low frequency by a canted surface-breaking crack. Stone et al. [10] used an approximation method to consider the diffraction of harmonic anti-plane shear waves by an edge crack normal to the free surface of a homogeneous semi-infinite space. Keer et al. [11] investigated the resonance effects for a crack near a free surface by determining displacement potentials that satisfy reduced wave equation and specified boundary conditions. The scattering of body waves by an inclined edge crack in a half-space has been studied by Zhang and Achenbach [12] by using boundary integral equations. Recently, by the method of matched asymptotic expansions, Abrahams and Wickham [13] obtained the dynamic stress field scattered by arbitrary shaped imperfections at the free surface of an isotropic half-space.

In this article, the method of the dislocation model [6] is extended to solve the problems of a finite crack with arbitrary angle in a semi-infinite space subjected to elastic SH-waves. The dynamic stress intensity factors for a given instance of time and various positions of cracks are of interest.

\section{FORMULATION}

Consider a rectangular Cartesian coordinate system located at the free surface of a semi-infinite space. A finite crack, shown in Fig. 1, can be located at an arbitrary position provided it is not in contact with the free surface. All lengths are normalized with respect to the half width of the crack length. Let the coordinate $x^{\prime} y^{\prime}$ rotate along with the crack, and its origin be at a distance 


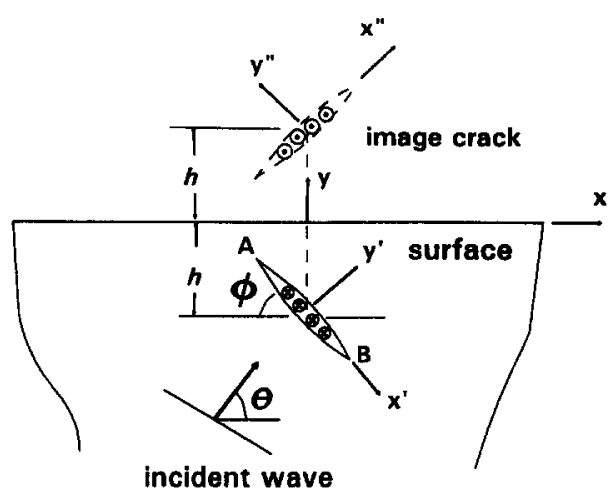

Fig. 1. Two arrays of vibration screw dislocations with the same but opposite density function.

$h$ below the surface. The crack occupies the region of $\left|x^{\prime}\right|<1, y^{\prime}=0,-\infty<z<+\infty$. The cracked elastic solid will undergo the anti-plane deformation if it is subjected to a harmonic anti-plane shear wave expressed as

$$
u_{z}^{(i)}=u_{o} \sin [\alpha(x \cos \theta+y \sin \theta)-\omega t]
$$

where $u_{o}$ is the displacement amplitude of the incident wave; $\theta$ is the angle of incidence; $\alpha=\omega / C_{t}$ is the wave number and $C_{t}$ the velocity of plane SH-waves. For a free boundary in the semi-infinite space, the reflected SH-wave which is in phase with the incident wave can be expressed as [14]

$$
u_{z}^{(r)}=u_{o} \sin [\alpha(x \cos \theta-y \sin \theta)-\omega t] .
$$

The superimposition of eqs (1) and (2) yields

$$
u_{z}=2 u_{o} \cos (\alpha y \sin \theta) \sin [\alpha x \cos \theta-\omega t] \text {. }
$$

The wave represents wave motion behaving as standing waves in the $y$ direction and progressive waves in the $x$ direction. For normal incident, $\theta=90^{\circ}$, the expression eq. (3) represents purely standing waves. Note that for $\theta=0^{\circ}$, i.e. for grazing incident, there is no reflected wave. Similarly, for a clamped surface in the semi-infinite solid, the superimposed wave can be obtained by employing the displacements vanishing at $y=0$ [14]. We have

$$
u_{z}=2 u_{o} \sin (\alpha y \cos \theta) \sin [\alpha x \cos \theta-\omega t] \text {. }
$$

In solid mechanics, the Green's function defines the response of the rigidly supported body to a point force or couple. For fracture problems dealing with static loads, the stress generated from each dislocation can be regarded as a Green's function. The elastic stress fields around loaded cracks, slip bands and twins, etc. have qualitatively similar features when viewed on a suitable scale. It is because they all represent the same type of incompatibility caused by the Somigliana dislocation. As has been proposed by Huang and So [6-8], this qualitative similarity between crack and dislocation causes the stress wave emitted from a vibrating screw dislocation to also be treated as a Green's function for elastodynamic crack problems. By superimposing an array of dislocations and adjusting the distribution density to fulfill the boundary conditions of traction-free of the crack faces, the stress intensity factors of cracks in an unbounded solid subjected to SH-waves were obtained.

A half-space concerning the two-dimensional motions in anti-plane shear is one of the simple problems that can be solved by the application of Green's function. The problem of a screw dislocation moving parallel to the surface with constant velocity and at a distance below the free surface had been solved by considering an image dislocation, with opposite sign, at the same distance above the free surface [15]. Based on the same concept, the results for a vibrating screw dislocation in an infinite medium can give an estimate of the stress field for a screw dislocation vibrating along the $x$-axis, and at a distance $h$ below the surface of the semi-infinite medium. Consider the incident sound wave propagated parallel to the negative $y$-direction, the screw dislocation with Burgers vector $(0,0, b)$, lying on the $z$-axis, then to oscillate in the $x$-direction. A SH-wave will emit, which can be represented by the Bessel functions given as [16] 


$$
\begin{aligned}
& \sigma_{y z}^{\infty}(x, y, t)=A \alpha^{2}\{a(y, r) \cos \omega t+b(y, r) \sin \omega t\} \\
& \sigma_{x z}^{\infty}(x, y, t)=A \alpha^{2}\{e(y, r) \cos \omega t+f(y, r) \sin \omega t\}
\end{aligned}
$$

where

$$
\begin{aligned}
a(y, r) & =\left(2 y 2 / r^{2}\right) J_{0}(\alpha r)+\left[(2 / \alpha r)-\left(4 y^{2} / \alpha r^{3}\right)\right] J_{1}(\alpha r) \\
b(y, r) & =\left(2 y^{2} / r^{2}\right) Y_{0}(\alpha r)+\left[(2 / \alpha r)-\left(4 y^{2} / \alpha r^{3}\right)\right] Y_{1}(\alpha r) \\
e(y, r) & =\left(2 x y / r^{2}\right) J_{0}(\alpha r)-\left(4 x y / \alpha r^{3}\right) J_{1}(\alpha r) \\
f(y, r) & =\left(2 x y / r^{2}\right) Y_{0}(\alpha r)-\left(4 x y / \alpha r^{3}\right) Y_{1}(\alpha r) \\
r^{2} & =x^{2}+y^{2} \\
A & =-b A_{0} \mu / 8
\end{aligned}
$$

where $A_{0}$ is the amplitude; $\omega$ is the vibrating frequency of the dislocation; $\mu$ is the shear modulus; the subscript $\infty$ denotes the dislocation in an infinite space. $J_{0}$ and $J_{1}$ are the Bessel functions of the first kind of order zero and one; $Y_{0}$ and $Y_{1}$ are the Bessel functions of the second kind of order zero and one, respectively. The problem is readily solved by superimposing the stress fields of the screw dislocation and its image; one obtains

$$
\sigma_{y z}(x, y, t)=\sigma_{y z}^{\infty}(x, h+y, t)-\sigma_{y z}^{\infty}(x, h-y, t) .
$$

When $y=0$, then $\sigma_{y z}=0$, as it should at the free surface. Based on the dislocation model [6-8], the crack can be simulated by superimposing an array of continuous distribution of vibrating infinitesimal screw dislocations at the position defined by $A_{0} \sin \left[\omega t+p\left(x^{\prime}\right)\right]$, where $A_{0}$ is assumed to be small; $p\left(x^{\prime}\right)$ is the phase lag compared with incident wave. The vibration frequency of the dislocations is assumed to be the same as the incident wave. Let $D\left(x^{\prime}\right)$ be the distribution function of the dislocation density. By assuming an array of image dislocations with density function $-D\left(x^{\prime}\right)$, the zero traction condition on the surface of the semi-infinite space is satisfied. The problem of diffraction of $\mathrm{SH}$-waves by a crack embedded in the semi-infinite space degenerates into diffraction of SH-waves, expressed in eq. (6), by two arrays of vibration screw dislocations with the same but opposite density functions in an infinite space shown in Fig. 1, i.e. an array of vibrating screw dislocations with it image dislocations in an infinite space. To fulfil the requirements of the equation of motion of each dislocation on the crack, $D\left(x^{\prime}\right)$ must satisfy the following equation for all points in the range of $-1<x^{\prime}<1$,

$$
\begin{aligned}
& \int_{-1}^{1} D\left(s^{\prime}\right) A \alpha^{2}\left\{c\left(x^{\prime}, s^{\prime}\right) \cos \left[\omega t+p\left(s^{\prime}\right)\right]+d\left(x^{\prime}, s^{\prime}\right) \sin \left[\omega t+p\left(s^{\prime}\right)\right]\right\} \mathrm{d} s^{\prime} \\
& \quad+\int_{-1}^{1}-D\left(s^{\prime \prime}\right) A \alpha^{2}\left\{\left[a\left(y^{\prime \prime}, R\right) \cos 2 \phi+e\left(y^{\prime \prime}, R\right) \sin 2 \phi\right] \cos \left[\omega t+p\left(s^{\prime \prime}\right)\right]\right. \\
& \left.\quad+\left[b\left(y^{\prime \prime}, R\right) \cos 2 \phi+f\left(y^{\prime \prime}, R\right) \sin 2 \phi\right] \sin \left[\omega t+p\left(s^{\prime \prime}\right)\right]\right\} \mathrm{d} s^{\prime \prime} \\
& =-2 \sigma_{o} \sin \theta \sin \left[\alpha\left(-h-x^{\prime} \sin \phi\right) \sin \theta\right] \sin \left(\alpha x^{\prime} \cos \phi \cos \theta-\omega t\right) \cos \phi \\
& \quad+2 \sigma_{o} \cos \theta \cos \left[\alpha\left(-h-x^{\prime} \sin \phi\right) \sin \theta\right] \cos \left(\alpha x^{\prime} \cos \phi \cos \theta-\omega t\right) \sin \phi
\end{aligned}
$$

where

$$
\begin{aligned}
c\left(x^{\prime}, s^{\prime}\right) & =J_{0}\left(\alpha\left|x^{\prime}-s^{\prime}\right|\right)+J_{2}\left(\alpha\left|x^{\prime}-s^{\prime}\right|\right) \\
d\left(x^{\prime}, s^{\prime}\right) & =Y_{0}\left(\alpha\left|x^{\prime}-s^{\prime}\right|\right)+Y_{2}\left(\alpha\left|x^{\prime}-s^{\prime}\right|\right) \\
R & =\left\{\left(2 h+x^{\prime} \sin \phi-s^{\prime \prime} \sin \phi\right)^{2}+\left(x^{\prime} \cos \phi-s^{\prime \prime} \cos \phi\right)^{2}\right\}^{1 / 2} \\
y^{\prime \prime} & =-\left[2 h \cos \phi+x^{\prime} \sin (2 \phi)\right]
\end{aligned}
$$

where $\sigma_{o}=\mu u_{o} \alpha$, denotes the value of the shear at the incident wave front and is taken finite as $\omega \rightarrow 0 . \phi$ is the inclined angle of the finite length crack. The unknown dislocation density $D(s)$ can be divided into two parts such as 


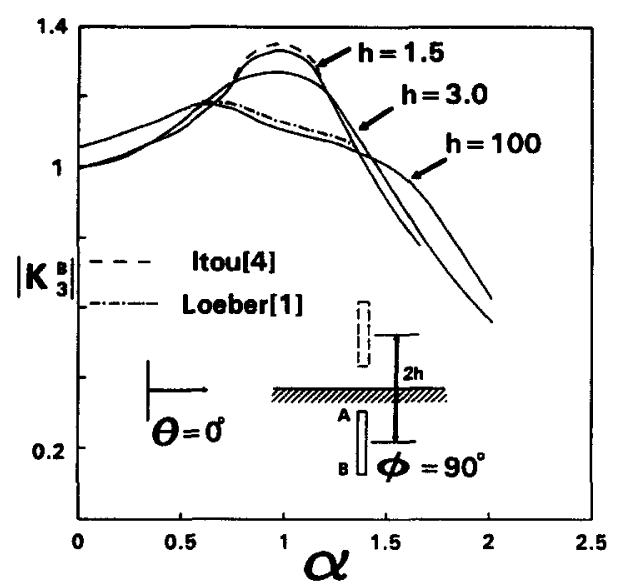

Fig. 2. The stress intensity factors for a vertical subsurface crack of finite length subjected to grazing incident waves.

$$
\begin{aligned}
& E_{1}(s)=A D(s) \cos [p(s)] \\
& E_{2}(s)=A D(s) \sin [p(s)] .
\end{aligned}
$$

Substituting eq. (8) into (7) and by comparing both sides, the coefficients of $\sin \omega t$ and $\cos \omega t$ in eq. (7) may be equated. A system of singular integral equations is obtained as

$$
\begin{aligned}
& \int_{-1}^{1} \alpha^{2}\left\{E_{1}\left(s^{\prime}\right)\left\{c\left(x^{\prime}, s^{\prime}\right)-\left[a\left(y^{\prime \prime}, R\right) \cos 2 \phi+e\left(y^{\prime \prime}, R\right) \sin 2 \phi\right]\right\}\right. \\
& \left.+E_{2}(s)\left\{d\left(x^{\prime}, s^{\prime}\right)-\left[b\left(y^{\prime \prime}, R\right) \cos 2 \phi+f\left(y^{\prime \prime}, R\right) \sin 2 \phi\right]\right\}\right\} \mathrm{d} s^{\prime} \\
= & -2 \sigma_{o} \sin \theta \sin \left[\alpha\left(-h-x^{\prime} \sin \phi\right) \sin \theta\right] \sin \left(\alpha x^{\prime} \cos \phi \cos \theta\right) \cos \phi \\
& +2 \sigma_{o} \cos \theta \cos \left[\alpha\left(-h-x^{\prime} \sin \phi\right) \sin \theta\right] \cos \left(\alpha x^{\prime} \cos \phi \cos \theta\right) \sin \phi \\
& \int_{-1}^{1} \alpha^{2}\left\{E_{1}\left(s^{\prime}\right)\left\{d\left(x^{\prime}, s^{\prime}\right)-\left[b\left(y^{\prime \prime}, R\right) \cos 2 \phi+f\left(y^{\prime \prime}, R\right) \sin 2 \phi\right]\right\}\right. \\
& \left.+E_{2}\left(s^{\prime}\right)\left\{-c\left(x^{\prime}, s^{\prime}\right)+\left[a\left(y^{\prime \prime}, R\right) \cos 2 \phi+e\left(y^{\prime \prime}, R\right) \sin 2 \phi\right]\right\}\right\} \mathrm{d} s^{\prime} \\
= & 2 \sigma_{o} \sin \theta \sin \left[\alpha\left(-h-x^{\prime} \sin \phi\right) \sin \theta\right] \cos \left(\alpha x^{\prime} \cos \phi \cos \theta\right) \cos \phi \\
& +2 \sigma_{o} \cos \theta \cos \left[\alpha\left(-h-x^{\prime} \sin \phi\right) \sin \theta\right] \sin \left(\alpha x^{\prime} \cos \phi \cos \theta\right) \sin \phi .
\end{aligned}
$$

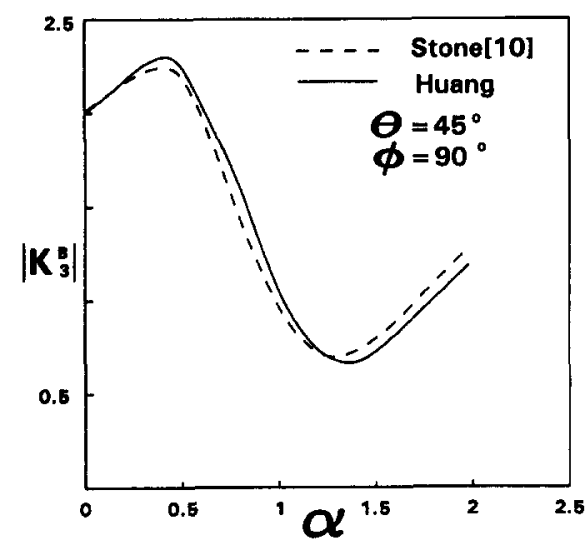

Fig. 3. Stress intensity factors for an edge crack normal to the free surface subjected to inclined incident waves. 


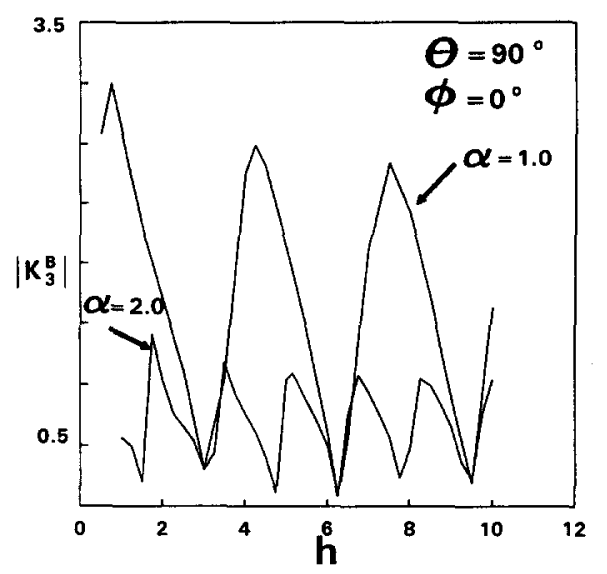

Fig. 4. Stress intensity factors of a horizontal subsurface crack vs distance $h$ below the free surface.

\section{NUMERICAL METHOD}

There are two unknowns, $E_{1}(s)$ and $E_{2}(s)$, to be determined in eq. (9) which can be separated into the singular parts, Hadamard's type and logarithmic singularity, and regular parts [6]. The solution of eq. (9) can be approximated by its truncated Chebyshev expansion [6].

$$
\begin{aligned}
& E_{1}(s) \approx \sum_{n=0}^{N} a_{n} U_{n}(s)\left(1-s^{2}\right)^{1 / 2} \\
& E_{2}(s) \approx \sum_{n=0}^{N} b_{n} U_{n}(s)\left(1-s^{2}\right)^{1 / 2} .
\end{aligned}
$$

Substituting eq. (10) into (9) and performing necessary integration procedures, the coefficients $a_{n}$, $b_{n}$ as the solutions of the equations can be obtained by using the Galerkin's method with $N=5$. Once the coefficients have been obtained, the dynamic stress intensity factor can be determined by carrying out the limiting process defined as

$$
\begin{aligned}
K_{3} & =\lim _{x \rightarrow 1}\left[2\left(x^{\prime}-1\right)\right]^{1 / 2}\left\{\int_{-1}^{1}\left[1 /(x-s)^{2}\right]\left[E_{1}(s) \sin \omega t+E_{2}(s) \cos \omega t\right] \mathrm{d} s\right\} \\
& =\left|K_{3}^{B}\right| \cos (\omega t-\delta)
\end{aligned}
$$

where $\left|K_{3}^{B}\right|$ is the amplitude of dynamic stress intensity factor with respect to tip $B ; \delta$ is the phase angle.

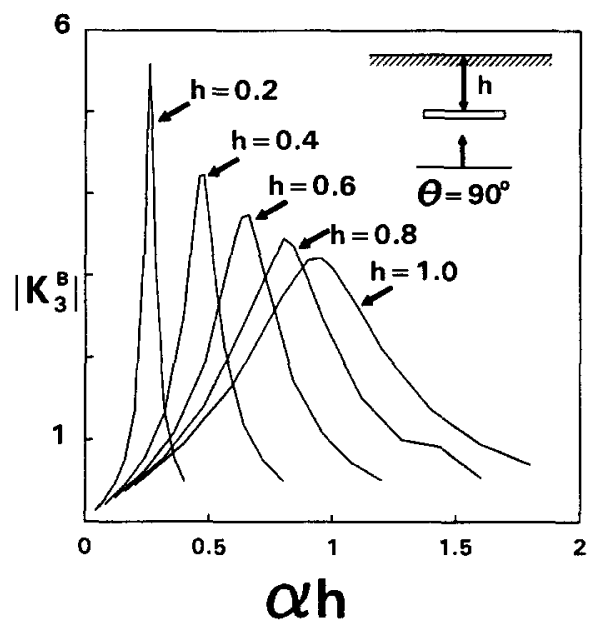

Fig. 5. Variation of stress intensity factors vs wave numbers and the distance between the free surface and crack. 


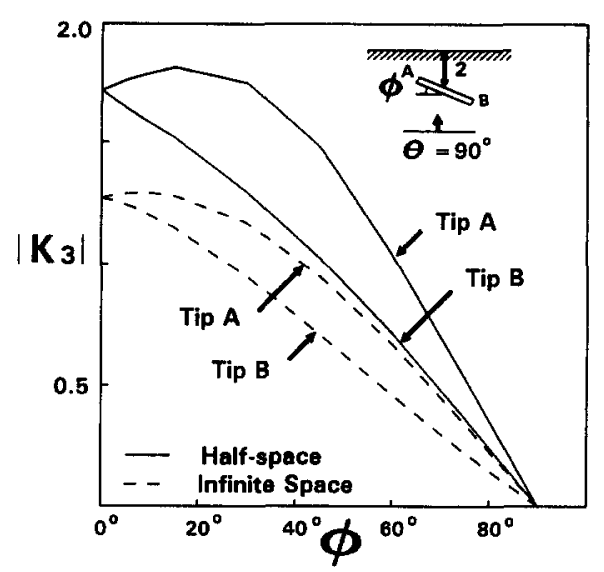

Fig. 6. Comparisons of the stress intensity factors of an inclined subsurface crack and an inclined crack embedded in an infinite medium.

$$
\begin{aligned}
\left|K_{3}^{B}\right| & =4\left\{\left[\sum_{n=0}^{N} a_{n}(n+1)\right]^{2}+\left[\sum_{n=0}^{N} b_{n}(n+1)\right]^{2}\right\}^{1 / 2} \\
\delta & =\tan ^{-1}\left\{\left[\sum_{n=0}^{N} a_{n}(n+1)\right]^{2} /\left[\sum_{n=0}^{N} b_{n}(n+1)\right]^{2}\right\}^{1 / 2} .
\end{aligned}
$$

Similarly, the amplitude of dynamic stress intensity factors with respect to the tip $A$ can be expressed as

$$
\left|K_{3}^{A}\right|=4\left\{\left[\sum_{n=0}^{N} a_{n}(-1)^{n}(n+1)\right]^{2}+\left[\sum_{n=0}^{N} b_{n}(-1)^{n}(n+1)\right]^{2}\right\}^{1 / 2}
$$

\section{RESULTS AND DISCUSSION}

In Fig. 2, the curves for $\theta=0^{\circ}$ and $\phi=90^{\circ}$ represent the stress intensity factors for collinear cracks of length 2 in an infinite space and agree with that given in ref. [4]. As the distance from crack to free surface becomes large enough, the dynamic stress intensity factors converge to the values of a single crack [1]. On the other hand, as one of the crack tips is nearly in contact with the free surface, the problem can be treated as the diffraction of elastic waves by an edge crack. The calculated stress intensity factors agree with the results of the approximation method derived by Stone et al. [10] as shown in Fig. 3. Note that due to the difference of the definition of the stress intensity factor, the calculated values are two times the values of Stone [10].

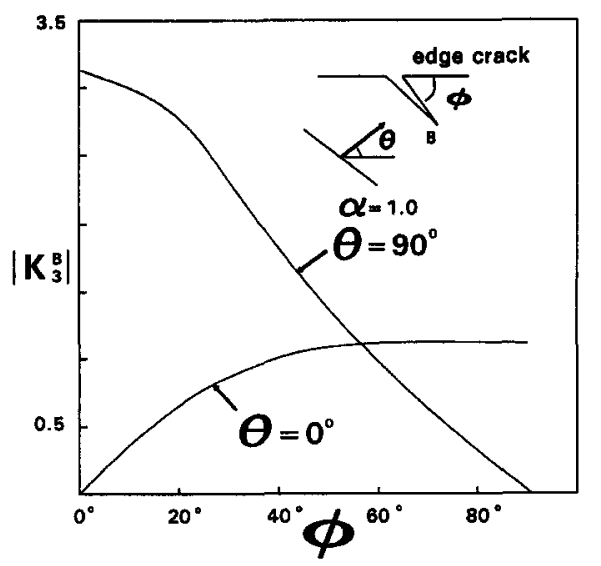

Fig. 7. The stress intensity factors for an inclined edge crack subjected to grazing and normal incident waves. 


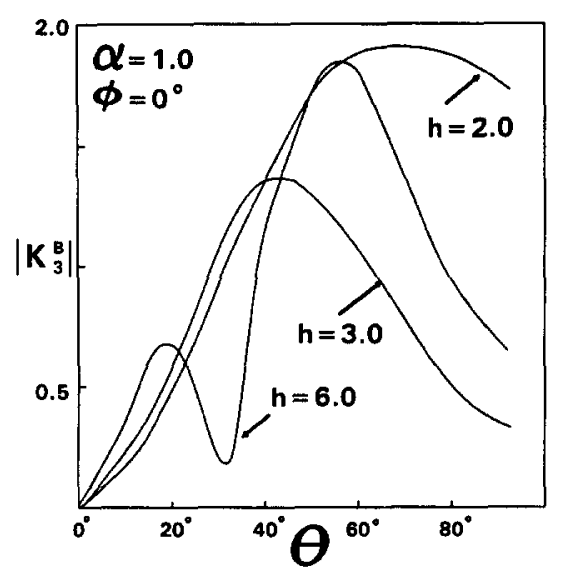

Fig. 8. Stress intensity factors of a horizontal subsurface crack vs the angle of incidence for traction-free surface.

To show the effect of the free surface, the stress intensity factors of a horizontal subsurface crack subjected to normal incident waves are shown in Fig. 4 . The stress waves are diffracted and reflected from boundary to boundary within the free surface and crack faces. The dynamic stress intensity factors fluctuate with the distance from crack to free surface at a nearly constant frequency which is proportional to the wave number. Resonance vibrations of the layer between the crack and the free surface are observed in Fig. 5. It is expected that a lower value of $h$ will produce a stronger resonance effect. Similar results had been shown in ref. [11] for considering a horizontal subsurface crack engulfed in an incident field of uniform tension and uniform shear. Results showing the variation of stress intensity factors vs the inclined angles of the subsurface crack subjected to normally incident waves are given in Fig. 6. The stress intensity factors of a crack embedded in an infinite medium are also presented for comparison. In general, due to the effect of the surface of the half-space, the stress intensity factor of a subsurface crack is larger than that of a crack in an infinite space. The stress intensity factors of an inclined edge crack are shown in Fig. 7. For grazing incident wave, the stress intensity factors increase with the inclined angle of the edge crack and become a constant when the inclined angle exceeds $60^{\circ}$.

Note that to fulfil the requirement of zero displacement on the clamped surface, the image dislocation with the same density function $D\left(x^{\prime}\right)$ and the superimposing wave expressed in eq. (4) should be applied. The difference between the numerical solutions of two types of boundary conditions, free of traction and clamped surface, is examined as shown in Figs 8 and 9, respectively. The effects of interface between the incident and reflected waves due to these two boundary conditions are quite different as can be seen from the calculated stress intensity factors of a

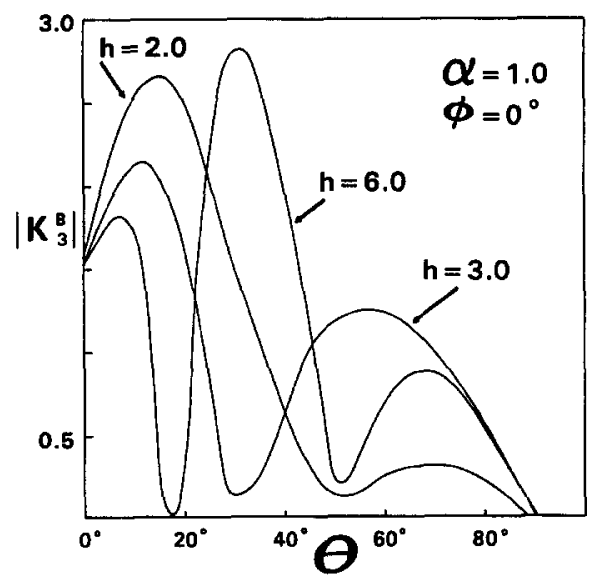

Fig. 9. Stress intensity factors of a horizontal subsurface crack vs the angle of incidence for clamped surface. 
horizontal subsurface crack subjected to incident waves with $\theta=0^{\circ}$ and $\theta=90^{\circ}$. For traction-free surface, the stress intensity factor is zero when the crack is subjected to a grazing incident wave. On the contrary, for clamped surface, the stress intensity factor is zero only when the crack is subjected to a normal incident wave. Furthermore, by comparing the amplitudes and fluctuations shown in Figs 8 and 9, the effect of the boundary of clamped type on the diffracted stress field seems to be more significant.

\section{CONCLUSIONS}

By combining the stress field emitted from an array of vibrating screw dislocations with its associated image array of dislocations, the stress intensity factors of a subsurface finite crack were determined. Furthermore, by assuming the crack to be nearly in contact with the free surface, the solution of the diffraction of elastic waves by an edge crack can be obtained. Conclusions from this work can be summarized below:

(1) The effect of the surface on the diffraction wave field is significant. The dynamic stress intensity factors fluctuate with the distance from crack to free surface at a nearly constant frequency which is proportional to the wave number. Resonance vibrations of the layer between the cracks and the free surface are observed as had been indicated in ref. [11].

(2) By assuming the crack to be nearly in contact with free surface, the problem of the diffraction of elastic waves by an edge crack can be solved by the dislocation model. For grazing incident wave, the stress intensity factors increase with the inclined angle of the edge crack and become a constant when the inclined angle exceeds $60^{\circ}$.

(3) The effects of interference between the incident and reflected waves can be quite different concerning the boundary condition. The results of two types of boundary conditions, free of traction and clamped surface, are compared.

Although the results presented here are for a single crack in a half-space, the method can be generalized to study the problem of crack-crack interaction under dynamic considerations by the technique presented in ref. [8]. Furthermore, the method of using dislocation model associated with image concept also shows a promising approach in handling more complex problems of diffraction of SH-waves by multiple cracks in a region bounded by two planes, for example a plate or a wave guide. These are presently under investigation and will be reported later.

\section{REFERENCES}

[1] J. F. Loeber and G. C. Sih, Diffraction of antiplane shear waves by a finite crack. J. Acoust. Soc. Am. 44, 90-98 (1968).

[2] A. K. Mal, Interaction of elastic waves with a Griffith crack. Int. J. Engng Sci. 8, 763-776 (1970).

[3] S. L. Jain and R. P. Kanwal, Diffraction of elastic waves by two coplanar Griffith cracks in an infinite elastic medium. Int. J. Solids Structures 8, 961-975 (1972).

[4] S. Itou, Diffraction of an anti-plane shear wave by two coplanar Griffith cracks in an infinite elastic medium. Int. J. Solids Structures 16, 1147-1153 (1980).

[5] K. Takakuda, Diffraction of plane harmonic waves by cracks. Bull. JSME 26, 478-493 (1983).

[6] J. Y. Huang and H. So, Determination of dynamic stress intensity factor by dislocation model. Int. J. Fracture 36, 187-198 (1988).

[7] J. Y. Huang and H. So, Diffraction of $P$ waves by two cracks at arbitrary position in an elastic medium. Engng Fracture Mech. 29, 335-347 (1988).

[8] H. So and J. Y. Huang, Determination of dynamic stress intensity factors of two finite cracks at arbitrary positions by dislocation model. Int. J. Engng Sci. 26, 111-119 (1988).

[9] S. K. Datta, Diffraction of SH-waves by an edge crack. J. appl. Mech. 46, 101-106 (1979).

[10] S. F. Stone, M. L. Ghosh and A. K. Mal, Diffraction of anti-plane shear waves by an edge crack. J. appl. Mech. 47, 359-362 (1980).

[11] L. M. Keer, W. Lin and J. D. Achenbach, Resonance effects for a crack near a free surface. J. appl. Mech. 51, 65-70 (1984).

[12] Ch. Zhang and J. D. Achenbach, Scattering of body waves by an inclined surface-breaking crack. Ultrasonics 26, $132-138$ (1988).

[13] I. D. Abrahams and G. R. Wickham, Scattering of elastic waves by an arbitrary small imperfection in the surface of a half-space. J. Mech. Phys. Solids 40, 1683-1706 (1992).

[14] J. D. Achenbach, Wave Propagation in Elastic Solids. North-Holland, New York, (1973).

[15] J. P. Hirth and J. Lothe, Theory of Dislocation. John Wiley, New York (1982).

[16] J. D. Eshelby, Dislocation as a cause of mechanical damping in metals. Proc. Roy. Soc. (Lond.) A197, 396-416 (1949). 